\title{
Spieltheorie: Spiel und Theorie für den Unterricht
}

\author{
"marcel.vorage@phsalzburg.at, PH Salzburg \\ eingereicht am: 04.02.2020, akzeptiert am: 13.08.2020
}

\begin{abstract}
Innovativität, zwischenmenschliche Interaktion und Zusammenarbeit sind wichtige Bestandteile von Innovationsprozessen. Aus humanistischen, emanzipatorischen und wirtschaftlichen Gründen ist es notwendig Schüler/innen dazu zu befähigen, erfolgreich an Innovationsprozessen teilzunehmen. Mit dem Ziel Innovativität und Zusammenarbeit zu fördern, wird in diesem Artikel die Spieltheorie herangezogen und auf Unterrichtskontexte übertragen. Der Artikel umfasst eine theoretische Einführung, spieltheoretische Beispiele im schulischen Kontext und konkrete Umsetzungsmöglichkeiten der Spieltheorie im Unterricht.
\end{abstract}

Keywords: Innovation, Schule, Spieltheorie, Interaktion, Zusammenarbeit

\section{Game Theory: Games and Theory for Secondary School}

Innovativeness, interaction and cooperation are integral parts of most innovative processes. Enabling pupils to participate more effectively in such processes is essential, from humanistic, emancipatory and economic viewpoints. This paper suggests using game-theory-tools to foster both innovativeness and cooperation. It provides a theoretical basis, examples of wellknown games in an educational context as well as specific ideas on how to successfully introduce game theory in class.

Keywords: innovation, school, game theory, interaction, cooperation

\section{$1 \quad$ Einleitung}

Unter Innovation versteht man eine neue Idee, die mit der Absicht verbunden ist, eine positive Veränderung für einzelne oder mehrere Personen herbeizuführen (Gryl 2013; Maier et al. 2001). Innovativität ist die Fähigkeit an Innovationsprozessen teilzunehmen (Weis et al. 2017). Lernende altersgerecht zur Innovativität zu befähigen, kann aus humanistischen, emanzipatorischen und wirtschaftlichen Perspektiven begründet werden. Die Teilnahme an Innovationsprozessen unterstützt die Selbständigkeit beim Denken und beim Handeln und befähigt zur erfolgreichen Selbstbestimmung (vgl. Scharf et al. 2017; Pike et al. 2016; Scharf et al. 2016; Love and Roper 2015; Zichy 2010).

Erfolgreiche Innovationsprozesse bestehen üblicherweise aus drei Teilbereichen, nämlich Reflexivität, Kreativität und Implementivität. Reflexivität ist die Fähigkeit, vorliegende Sachverhalte und (eigenes) Verhalten zu hinterfragen. Kreativität ist die Fähigkeit, neue Ideen und Lösungen zu entwickeln.
Implementivität ist die Fähigkeit, andere zu überzeugen und einzubinden. Es ist dabei nicht notwendig, alle Teilbereiche in einer bestimmten Reihenfolge zu durchlaufen. In der Schule muss die Förderung von Innovativität dementsprechend nicht zwangsläufig alle Teilbereiche umfassen, da sich Lernende nicht alle Teilbereiche (gleichzeitig) aneignen müssen (vgl. Jekel et al. 2015; Weis et al. 2017; Scharf et al. 2018). Im Unterricht können zum Beispiel kreative Denkstrategien vermittelt (Michalko 2006) oder innovative Unterrichtmethoden eingesetzt werden (vgl. Seitz 2008; Ebner 2005), um die Innovativitäts-Fähigkeit der Lernenden zu fördern.

Innovation ist ein sozialer Prozess, und es ist unwahrscheinlich, dass eine einzelne Person oder ein einzelner Betrieb alle Phasen eines Innovationsprozesses erfolgreich meistern kann (vgl. Gryl 2013; Bornemann 2011; De Faria et al. 2010; Franke 2005; Pittaway et al. 2004). Dementsprechend ist die zwischenmenschliche Interaktion ein bedeutender Bestandteil von Innovationsprozessen. Der Artikel umfasst eine Auswahl von vereinfachten spieltheore- 
tischen Darstellungen zwischenmenschlicher Interaktionen, die gut dafür geeignet sind, Unterschiede zwischen diesen dargestellten Interaktionsformen zu erkennen. Das Ziel dieses Artikels ist es, Schüler/innen darauf aufmerksam zu machen, dass zwischenmenschliche Interaktion in unterschiedlichen Kontexten $\mathrm{zu}$ unterschiedlichen Ergebnissen führen kann. So kann in spezifischen Fällen die Verfolgung der eigenen Ziele zu einem bestmöglichen Ergebnis führen, während unter anderen Umständen ein solches Ergebnis nur durch frühzeitige Zusammenarbeit und Abstimmung erreicht werden kann. Zudem gibt es Situationen, in denen weder Zusammenarbeit und Abstimmung, noch die Verfolgung der eignen Ziele ausreichen, um ein für alle zufriedenstellendes Ergebnis zu erreichen (vgl. Dixit et al. 2014; Fudenberg \& Tirole 1991; Gibbons 1992; Tadelis 2013; Watson 2013; Von Neumann \& Morgenstern 1944).

$\mathrm{Da}$ die Bewertung der zwischenmenschlichen Interaktion subjektiv ist, ist diese durch die Teilnehmer/ innen bestimmt. Somit sind nicht nur finanzielle oder materielle Ergebnisse entscheidend, sondern auch soziale und emotionale Bewertungen. Im Bereich der Innovation sind folglich vielfältige persönliche Bewertungsgrundlagen für Interaktionen entscheidend. Somit sind neben finanziellen oder materiellen Erträgen auch positive Gefühle, die die Interaktionen begleiten können (z. B. Vertrauen, Freude am Austausch, Zugehörigkeitsgefühl etc.), wichtig für die Bewertung der Ergebnisse (vgl. Franke \& Shah 2003; Lakhani \& Wolf 2003; Lerner \& Tirole 2002; Landry et al. 2001).

Wenn Schulen es schaffen, kreative Denkprozesse anzuregen, zwischenmenschliche Abhängigkeiten aufzuzeigen und die persönliche Bewertung von Zusammenarbeit bei innovativen Prozessen zu steigern, dann leisten diese nicht nur einen wichtigen Beitrag für die Bildung ihrer Schüler/innen, sondern auch für (zukünftige) Innovationsprozesse. Die Unterrichtsidee in diesem Artikel bietet dafür erste Umsetzungsvorschläge, wie der Ansatz der Spieltheorie die Erreichung dieser Bildungsziele in spielerischer Art und Weise unterstützen kann. Spieltheorie wurde in der Schule als Methode in der Mathematik vorgeschlagen und erfolgreich umgesetzt (Ablinger \& Hauer-Typpelt 2008; Girnat 2010; Schneeberger 2018). Auch im Netz befindet sich zumindest ein Unterrichtsbeispiel (Lehrer-Online 2020).

Im Artikel wird zuerst die notwendige spieltheoretische Basis gelegt. Diese Basis wird mit fünf klassischen Spielen aus der Spieltheorie ergänzt, wobei jedes dieser Spiele mit einem Beispiel aus der Schule und einem Beispiel mit Innovationsbezug versehen ist. Daran anschließend folgt eine Unterrichtsidee, die Spieltheorie mit Innovation und deren Teilbereichen Reflexivität, Kreativität und Implementivität verbindet.

\section{Zwischenmenschliche Interaktion in der Spieltheorie}

Die Spieltheorie modelliert zwischenmenschliche Interaktion als Spiel. Teilnehmer/innen an einem Spiel in der Spieltheorie werden begrifflich als "Spieler" bezeichnet, ob männlich oder weiblich. Spieler treffen Entscheidungen, deren Folgen von den Entscheidungen der anderen Spieler abhängen. Demzufolge machen die Spieler sich im Vorhinein Gedanken darüber, wofür sich andere Spieler entscheiden könnten (Von Neumann 1928; Von Neumann \& Morgenstern 1944).

\section{Interaktion (= Spiel) bedeutet in der Spieltheorie:}

- Es gibt (mindestens) zwei Spieler.

- Jeder Spieler versucht das beste Ergebnis für sich zu erreichen.

- Ergebnisse können von Spielern nach Vorlieben gereiht werden, d. h. die Ergebnisse sind ordinal.

- Die Bewertung der eigenen Ergebnisse ist persönlich und subjektiv. Die Einschätzungen der Spieler können neben finanziellen oder materiellen Ergebnissen auch soziale und emotionale Aspekte beinhalten.

- Jeder Spieler entscheidet sich zwischen (mindestens) zwei Möglichkeiten (oder Optionen).

- Die Entscheidung von einem Spieler beeinflusst das Ergebnis von (mindestens) einem anderen Spieler.

\section{Die Spiele in diesem Artikel}

Dieser Artikel befasst sich mit einer beschränkten Auswahl von Spielen der Spieltheorie. Um einen leichten Einstieg zu ermöglichen, sind hier nur Spiele mit zwei Spielern angeführt, die jeweils zwischen zwei (oder drei) Möglichkeiten entscheiden. Die Spieler entscheiden sich gleichzeitig für eine ihrer Möglichkeiten. Sie können die Möglichkeiten nicht austauschen oder eine Mischform aus zwei Entscheidungen wählen. Die Spieler entscheiden vorerst unabhängig voneinander, d.h. ohne ihre jeweilige Entscheidung miteinander abzustimmen. Die Auswirkungen einer Abstimmung ihrer Entscheidungen durch Zusammenarbeit werden erst im Anschluss diskutiert.

\section{Die theoretischen Grundlagen der Spiele}

\section{Die Darstellung von Spielen mit zwei Spielern}

Die Ergebnisse eines Spiels können in zwei Formen dargestellt werden: in einem Spielbaum oder einer Extensivform (Abb. 1a) und einer Normalform 

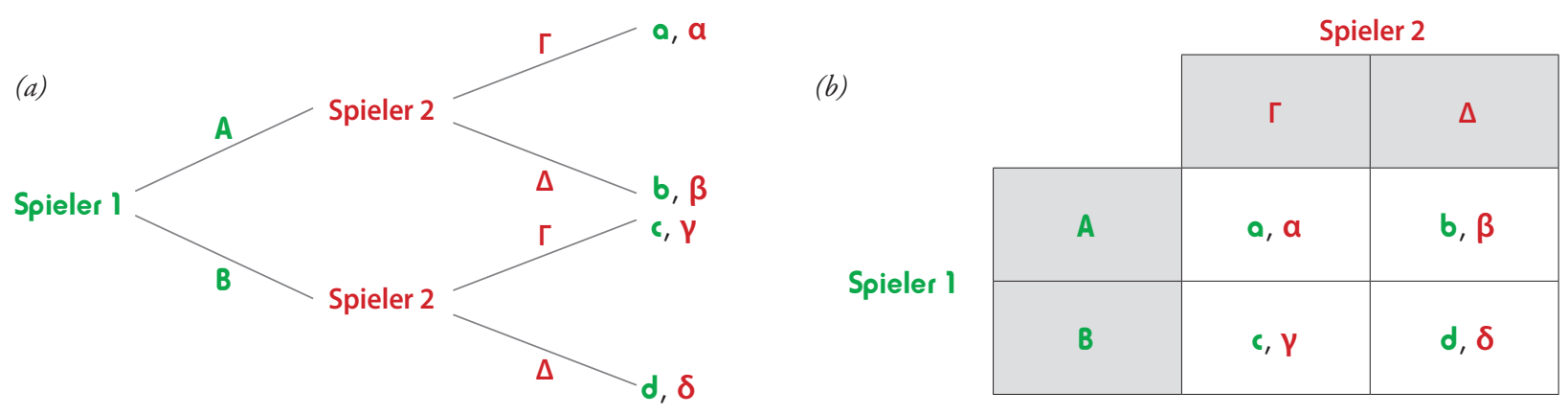

Abb. 1: Darstellung eines Spiels in (a) Spielbaum und (b) Normalform (eigene Darstellung).

(Abb. 1b). In jeder der Darstellungen passiert das Gleiche. Spieler 1 entscheidet sich zwischen den Optionen $A$ und $B$ und kommt zum Ergebnis $a, b$, c oder d. Spieler 2 entscheidet sich zwischen den Optionen $\Gamma$ und $\Delta$ und kommt zum Ergebnis $a, \beta$, $\gamma$ oder $\delta$. Trotz der Darstellung im Spielbaum, wählen beide Spieler gleichzeitig die von Ihnen bevorzugte Option. Es gibt demzufolge einen zweiten äquivalenten Spielbaum, wobei die Reihenfolge der Spieler in der Darstellung vertauscht wird.

Wenn Spieler 1 sich für Option A entscheidet und Spieler 2 sich für Option 「 entscheidet, dann erlangt Spieler 1 das Ergebnis a und Spieler 2 das Ergebnis a (in der Kurzform a, a). Wenn Spieler 1 sich für Option $A$ entscheidet und Spieler 2 sich für Option $\Delta$ entscheidet, dann erlangt Spieler 1 das Ergebnis b und Spieler 2 das Ergebnis $\beta$ (in der Kurzform b, $\beta$ ), usw.

Abhängig davon wie Spieler 1 die Ergebnisse $a, b, c$ und $d$ nach Präferenz und Spieler 2 die Ergebnisse $a$, $\beta, \gamma$ und $\delta$ nach Präferenz reiht, ergeben sich Spiele mit anderen Eigenschaften und Entscheidungsdynamiken. Diese Reihung der Ergebnisse nach Präferenz ist sehr wichtig, da sie die Entscheidungen der beiden Spieler im Spiel bestimmt.

In allen noch folgenden Beispielen werden Zahlenwerte für $a, b, c$ und $d$ sowie für $a, \beta, \gamma$ und $\delta$ eingesetzt. Diese Zahlenwerte reflektieren die Reihung der Ergebnisse durch beide Spieler und sind im Artikel so einfach wie möglich $(0,1,2,3)$. Damit wird es leichter die Eigenschaften eines bestimmten Spiels zu erkennen, die Optionen zu vergleichen und diese nach Präferenz zu reihen (höher ist besser). Weil es vier

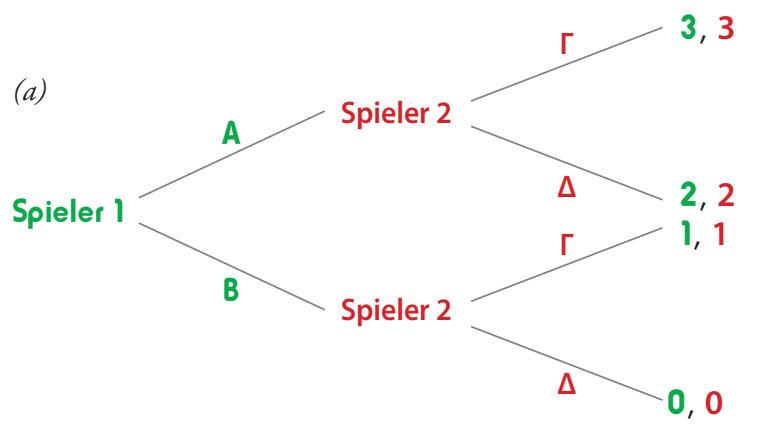

mögliche Kombinationen von Entscheidungen gibt, reichen diese vier Zahlen zur Reihung aller möglichen Präferenzen. Wenn es aber zusätzliche Optionen (oder Spieler) gibt, dann sind weitere nichtnegative ganze Zahlen notwendig, um alle möglichen Reihungen vorzunehmen.

\section{Dominante Strategien und (Nash-)Gleichgewicht an einem Beispiel}

Ein Gleichgewicht ist eine Kombination von Entscheidungen, wobei kein Spieler im Nachhinein die getroffene Entscheidung verändern möchte. Das heißt: „Jetzt wo ich die Entscheidung des anderen Spielers kenne, bleibe ich bei meiner Entscheidung."

Gibt es Gleichgewichte im Spiel von Abb. 2? Die Ergebnisse und Entscheidungsmöglichkeiten der Spieler sehen im Detail folgendermaßen aus:

- A, $\Gamma$ (ganz oben im Spielbaum und linksoben in der Normalform): Spieler 1 hat keinen Grund die Entscheidung zu ändern. Option $A$ ist besser als Option B, weil $3>1(a>c)$. Spieler 2 hat auch keinen Grund die Entscheidung zu ändern. Option $\Gamma$ ist besser als Option $\Delta$ weil $3>2(\alpha>\beta)$. Demzufolge ist $A, \Gamma$ ein Gleichgewicht.

- A, $\Delta$ (an zweiter Stelle im Spielbaum und rechtsoben in der Normalform): Spieler 1 hat keinen Grund die Entscheidung zu ändern. Option A ist besser als Option B, weil $2>0(b>d)$. Spieler 2 sollte die Entscheidung ändern. Option $\Delta$ ist schlechter als Option $\Gamma$, weil $2<3(\beta<\alpha)$. Demzufolge ist $A, \Delta$ kein Gleichgewicht.

(b)

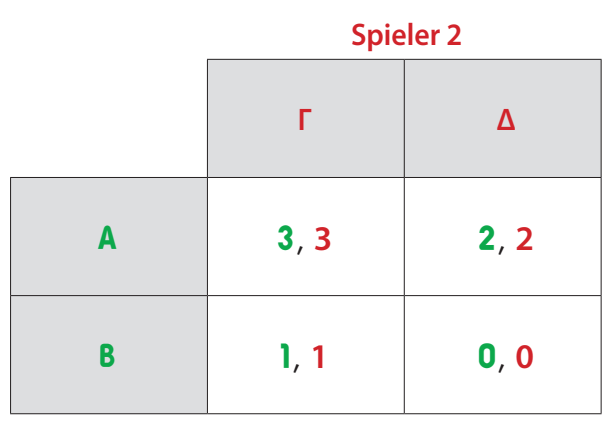

Abb. 2: Darstellung eines beispielhaften Spiels in (a) Spielbaum und (b) Normalform (eigene Darstellung). 
- B, Г (an dritter Stelle im Spielbaum und linksunten in der Normalform): Spieler 1 sollte die Entscheidung ändern. Option B ist schlechter als Option A, weil $1<3(c<0)$. Spieler 2 hat keinen Grund die Entscheidung zu ändern. Option $\Gamma$ ist besser als Option $\Delta$, weil $1>0(\gamma>\delta)$. Demzufolge ist $B, \Gamma$ kein Gleichgewicht.

- B, $\Delta$ (ganz unten im Spielbaum und rechtsunten in der Normalform): Spieler 1 sollte die Entscheidung ändern. Option B ist schlechter als Option A, weil $0<2(d<b)$. Auch Spieler 2 sollte die Entscheidung ändern. Option $\Delta$ ist schlechter als Option $\Gamma$, weil $0<1(\delta<\gamma)$. Demzufolge ist $B, \Delta$ kein Gleichgewicht.

Die Option A wird der Option B vorgezogen, wenn Option A für den entscheidenden Spieler besser als Option B ist, unabhängig davon, wofür sich der andere Spieler entscheidet. Ist das der Fall, so fällt Option $\mathrm{B}$ weg. Es gibt nämlich keinen Grund Option B zu wählen. Das bezeichnet man als dominante Strategie: Option A ist immer besser als (bzw. dominiert) Option B. Durch das Wegfallen von Option B vereinfacht sich das Spiel und es sind weitere dominante Strategien möglich (= iterative Dominanz). Das heißt: „Diese Option wähle ich ohnehin nicht, weil eine andere immer besser für mich ist."

Gibt es dominante Strategien im Spiel von Abb. 2? Im Detail sieht es folgendermaßen aus:

- Spieler 1: $3>1(a>c)$ und $2>0(b>d)$ bedeutet, dass Option A immer besser als Option B ist, unabhängig davon, ob sich Spieler 2 für $\Gamma$ oder $\Delta$ entscheidet. Demzufolge ist die Entscheidung für Option A die dominante Strategie für Spieler 1. In der Abbildung 3 fällt demnach Option B weg.

- Spieler 2: $3>2(\alpha>\beta)$ und $1>0(\gamma>\delta)$ bedeutet, dass Option $\Gamma$ immer besser als Option $\Delta$ ist, unabhängig davon, ob sich Spieler 1 für $A$ oder $B$ entscheidet. Demzufolge ist für Spieler 2 die Entscheidung für Option $\Gamma$ die dominante Strategie. In Abbildung 3 fällt dadurch Option $\Delta$ weg.

Ein Nash-Gleichgewicht ist jenes Gleichgewicht, dass über iterative Dominanz auch tatsächlich durch

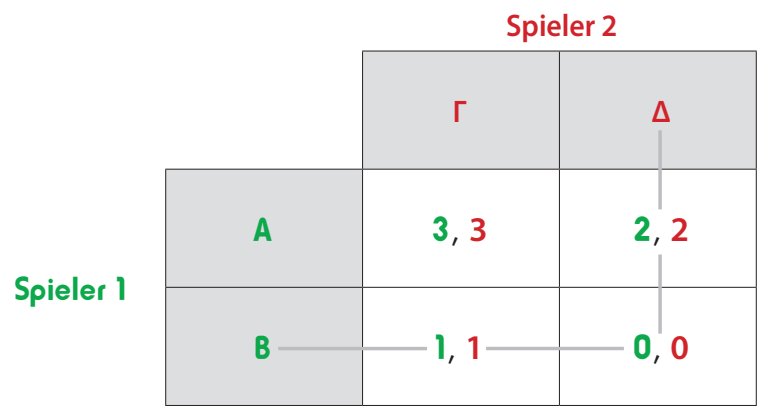

Abb. 3: Beispiel mit dominante Strategien die Spieler gewählt wird. In diesem Spiel ist A, Г das Nash-Gleichgewicht. Die iterative Dominanz ergibt A, Г. Im Nachhinein möchte auch keiner der Spieler von der Entscheidung A, $\Gamma$ abweichen.

\section{Fünf klassische Beispiele aus der Spiel- theorie im schulischen und innovativen Kontext}

In diesem Absatz werden fünf klassische Spiele der Spieltheorie mit einem Beispiel aus der Schule erklärt. Ein möglicher Bezug zur Lebenswelt der Schüler/ innen wird damit ersichtlich. Zusammen mit einem weiteren Beispiel zur Innovation macht es Möglichkeiten zum mündigen Handeln ersichtlich. Die innovationsbezogenen Beispiele befinden sich an verschiedenen Schnittstellen mit Gesellschaft, Wirtschaft, Politik und Umwelt. Kurz werden auch die namensgebenden ,Originalbeispiele' skizziert (vgl. Rousseau 1755; Kuhn \& Tucker 1950; Von Neumann \& Morgenstern 1944).

Es werden dabei die Gleichgewichte, dominanten Strategien und Nash-Gleichgewichte angegeben. In jedem Spiel haben die Ergebnisse andere Verhältnisse zueinander. Die Ergebnisse sind in den Beispielen auf nichtnegative ganzen Zahlen reduziert und damit einfach in ihrer Präferenz zu reihen. Für die Darstellung der Spiele wird die Normalform verwendet. Diese Spiele sind mit unterschiedlichen Zahlenwerten an verschiedenen Stellen in spieltheoretischen Textbüchern und grundlegenden wissenschaftlichen Artikeln auffindbar (vgl. Dixit et al. 2014; Fudenberg \& Tirole 1991; Gibbons 1992; Kuhn \& Tucker 1950; Tadelis 2013; Watson 2013; Von Neumann \& Morgenstern 1944).

\section{Deadlock}

- Schule: Zwei Schüler/innen stellen jeweils eine unterschiedliche Frage zum Unterricht. Die begrenzte Zeit der Lehrperson wird gleichmäßig auf deren Beantwortung aufgeteilt. Umso mehr Zeit die Lehrperson für eine Frage hat, umso besser

\begin{tabular}{|c|c|c|c|}
\hline & \multicolumn{2}{|c|}{ Schüler/in 2} \\
\hline & & $\begin{array}{l}\Gamma: \text { Nicht } \\
\text { Fragen }\end{array}$ & $\Delta:$ Fragen \\
\hline \multirow{2}{*}{ Schüler/in 1} & $\begin{array}{l}\text { A: Nicht } \\
\text { fragen }\end{array}$ & 1,1 & 0,3 \\
\hline & B: Fragen & 3,0 & 2,2 \\
\hline
\end{tabular}

Abb. 4: Beispiel für „Deadlock“ 
wird die Frage beantwortet. Ohne Fragen geht der Unterricht ohne Unterbrechungen weiter.

- Innovation: Zwei Personen können jeweils eine Verleihfirma für E-Roller in einer Stadt gründen. Es kann eine (Option B oder $\Delta$ ) oder keine (Option $A$ oder $\Gamma$ ) Firma von jedem gegründet werden. Gründen beide eine Firma, dann wird der Markt geteilt. Wird nur eine Firma gegründet, dann ist der Gründer Monopolist in der Stadt, während die andere Person sich an der Anwesenheit der E-Roller im Verkehr und im Straßenbild ärgert. Wird keine Firma gegründet, dann bleiben zumindest der Straßenverkehr und die Stadt weitgehend von E-Rollern verschont.

- Das Original: Die zwei Spieler können entweder zusammenarbeiten (Option A oder $\Gamma$ ) oder abtrünnig werden (Option B oder $\Delta$ ). Am liebsten ist ein Spieler abtrünnig, während der Mitspieler zusammenarbeitet.

- Eigenschaften:
- Gleichgewicht: B, $\Delta$
- Dominant: $B$ und $\Delta$
- Nash-Gleichgewicht: B, $\Delta$

\section{Kampf der Geschlechter (Battle of the sexes)}

- Schule: Zwei Schüler/innen müssen für GW und Mathematik lernen. Sie können entweder ihre Unterlagen für GW oder Mathe zum gemeinsamen Lernen mitbringen. Wenn beiden die gleichen Unterlagen mitbringen, können sie sich gegenseitig helfen. Schüler/in 1 mag lieber GW lernen, Schüler/in 2 mag lieber Mathe lernen.

- Innovation: In einer Straße in Wien soll eine kühle Zone entstehen. Es gibt zwei potentielle Standorte. Zwei Bewohner/innenvertreter sind für die grüne Zone, aber Sie bevorzugen den Standort vor ihrem Haus. Nur wenn beide Vertreter den gleichen Standort wählen, wird die grüne Zone verwirklicht.

- Das Original: Eine Frau (Schüler/in 1) und ein Mann (Schüler/in 2) wollen den Abend gemeinsam verbringen und haben sich verabredet. Leider wissen beide nicht mehr, ob sie sich jetzt bei der

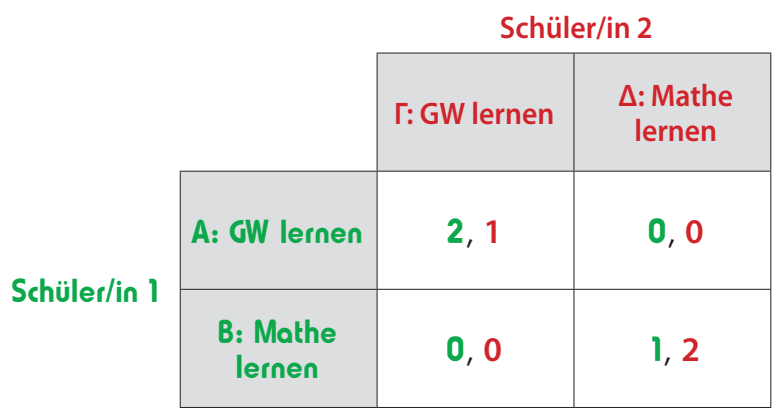

Abb. 5: Beispiel für „Kampf der Geschlechter“
Oper (Vorliebe Frau, Option A oder $\Gamma$ ) oder beim Boxwettkampf (Vorliebe Mann, Option B oder $\Delta)$ treffen sollen. Das wichtigste ist, den Abend gemeinsam zu verbringen, aber am liebsten dort, wo die eigene Vorliebe liegt. Beide entscheiden sich unabhängig voneinander wohin sie gehen, zur Oper oder zum Boxwettkampf.

- Eigenschaften:
- Gleichgewichte: A, $\Gamma$ und B, $\Delta$
- Dominant: -
- Nash-Gleichgewicht: -

\section{Hirschjagd (Stag hunt)}

- Schule: Zwei Schüler/innen schreiben gemeinsam eine schriftliche Arbeit. Sie teilen sich die Arbeit auf und machen ihren Teil dann jeweils zu Hause. Nur wenn beide hart dafür arbeiten, können sie ein sehr gutes Ergebnis erzielen. Wenig Einsatz von beiden Schülern/Schülerinnen ergibt eine Arbeit, die gerade noch für ein Genügend reicht. Arbeitet nur eine/ $\mathrm{r}$ der beiden Schüler/innen hart, liegt das Ergebnis dazwischen.

- Innovation: Zwei Designer/innen entwickeln gemeinsam eine Modelinie. Nur wenn beide hart dafür arbeiten, entwerfen sie Kleidung, die bald der Trend wird. Wenig Einsatz von beiden resultiert in einer weiteren Modelinie, die sich nicht von anderen unterscheidet. Arbeitet nur eine/r der beiden Designer/innen hart, ist die Kleidung zwar ausgefallen, einen Trend löst es aber nicht aus.

- Das Original: Zwei Jäger/innen können entweder ein Hirsch jagen (Option A oder $\Gamma$ ) oder ein Kaninchen (Option B oder $\Delta$ ). Um bei der Hirschjagd erfolgreich zu sein, müssen beide Jäger/innen zur Hirschjagd antreten. Wird ein Hirsch oder ein Kaninchen getötet, dann wird die Beute von den Jägern/Jägerinnen verspeist, die bei der erfolgreichen Jagd beteiligt waren.

- Eigenschaften:

- Gleichgewichte: A, Г und B, $\Delta$

- Dominant: -

- Nash-Gleichgewicht: -

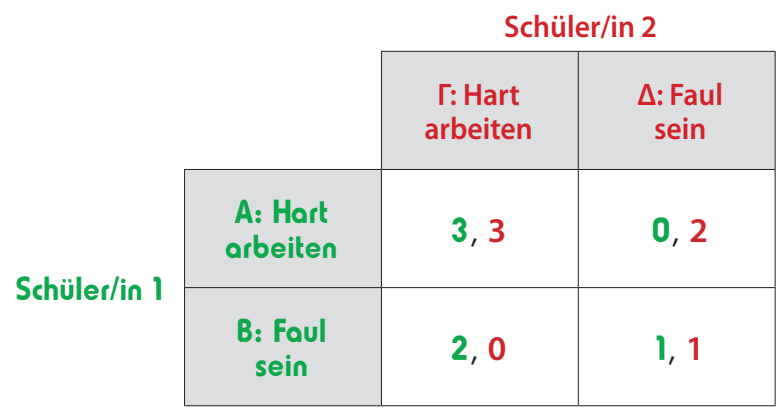

Abb. 6: Beispiel für „Hirschjagd“ 
Anmerkung: Obwohl die Eigenschaften gleich sind wie beim Kampf der Geschlechter, ist die Reihung der Präferenzen unterschiedlich. Bei der Hirschjagd bevorzugen beide Spieler das Ergebnis der erfolgreichen Hirschjagd über das Ergebnis der erfolgreichen Kaninchenjagd. Beim Kampf der Geschlechter bevorzugt die Frau den gemeinsamen Besuch der Oper, der Mann den gemeinsamen Besuch des Boxwettkampfs.

\section{Gefangenendilemma (Prisoner's Dilemma)}

- Schule: Es besteht der Verdacht, dass abgeschrieben wird, weil zwei Schüler/innen sehr ähnliche Arbeiten abgegeben haben. Die beiden Schüler/ innen werden einzeln befragt. Es kann entweder der/ die Andere verpetzt oder nicht verpetzt werden. Wenn keine/r der beiden petzt, gibt es eine Verwarnung für beide Schüler/innen. Wenn ein/e Schüler/in den Anderen verpetzt, kommt diese(r) Schüler/in ohne Verwarnung davon, während der / die verpetzte Schüler/in schwer bestraft wird. Petzen beide, dann gibt es für beide eine Strafe.

- Innovation: In zwei Laboren wird nach einem Impfstoff gegen ein neues Virus geforscht. Labore können ihre Forschungsergebnisse teilen (Option A oder $\Gamma$ ) oder geheim halten (Option B oder $\Delta$ ). Teilen beide Labore ihre Forschungsergebnisse, dann gibt es einen effektiveren Impfstoff. Beide Labore teilen den Ruhm und finanzielle Gewinne. Teilt nur eines der beiden Labore, dann geht das andere Labor leer aus und dann erforscht das geheim haltende Labor alleine an einem effektiveren Impfstoff. Halten beide Labore ihre Forschung geheim, dann gibt es nur einen bedingt effektiven Impfstoff.

- Das Original: Zwei Kriminelle werden gleichzeitig verhaftet und getrennt voneinander befragt. Wenn einer der Kriminellen redet (Option B oder $\Delta$ ) und dabei seinen Komplizen belastet, gibt es dafür eine Strafmilderung. Der Komplize bekommt dann eine höhere Strafe. Wenn beiden schweigen (Option A und $\Gamma$ ), werden beiden vergleichsweise leicht bestraft.

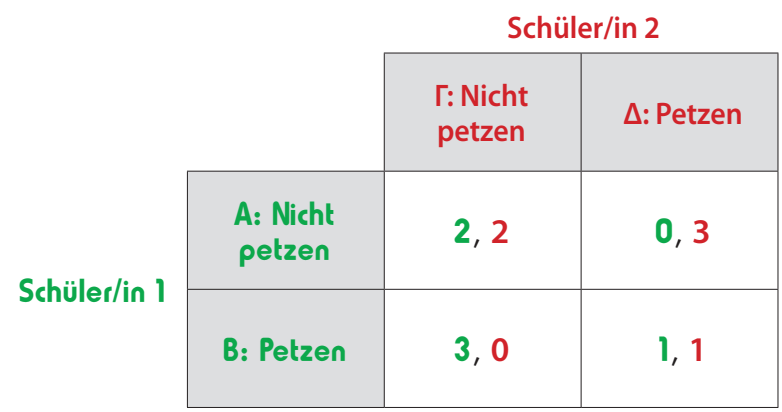

Abb. 7: Beispiel für „Gefangenendilemma“
- Eigenschaften:

- Gleichgewichte: B, $\Delta$

- Dominant: $B$ und $\Delta$

- Nash-Gleichgewicht: B, $\Delta$

Anmerkung: A, $\Gamma$ enthält für beide Schüler/innen ein besseres Ergebnis als das Nash-Gleichgewicht $B, \Delta$. Es wird durch die iterative Dominanz aber ausgeschlossen. Das heißt, wenn jeder in diesem Spiel die individuell optimale Entscheidung trifft, kommt ein für beide suboptimales Ergebnis zustande.

\section{Kopf oder Zahl mit zwei Münzen (Matching pennies)}

- Schule: Im Sportunterricht wird beim Fußballspielen ein Elfmeter vergeben. Schüler/in 1 (Torwart) und Schüler/in 2 (Schütze) müssen sich entweder für das linke (Option A oder $\Gamma$ ) oder rechte Eck (Option B oder $\Delta$ ) entscheiden. Wenn Torwart und Schütze das gleiche Eck wählen, wird der Ball abgefangen. Wählen sie jeweils ein anderes Eck, ist der Ball im Tor. Der Torwart gewinnt, wenn der Ball abgefangen wird, der Schütze, wenn der Ball ins Tor geht.

- Innovation: Bei immer weiter eskalierenden Protesten gegen Polizeigewalt stehen Demonstranten und die Polizei einander gegenüber. Die Demonstranten (Schüler/in l) können zum Protest entweder ein Schild (A) oder einen Laubbläser (B) mitbringen. Die Polizei (Schüler/in 2) kann Schlagstöcke $(\Gamma)$ oder Tränengas $(\Delta)$ einsetzen. Haben Demonstranten das richtige mit, dann bekommen sie von den Entscheidungsträgern Zugeständnisse. Haben sie das Falsche mit, müssen sie aufgeben.

- Das Original: Jeder der zwei Spieler hat eine Münze und legt diese mit Kopf oder Zahl nach oben auf den Tisch. Ein Spieler (Schüler/in 1) gewinnt, wenn beide Münzen die gleiche Seite oben haben. Ein Spieler (Schüler/in 2) gewinnt, wenn beide Münzen eine unterschiedliche Seite oben haben.

- Eigenschaften:

- Gleichgewicht: -

- Dominant: -

- Nash-Gleichgewicht: -

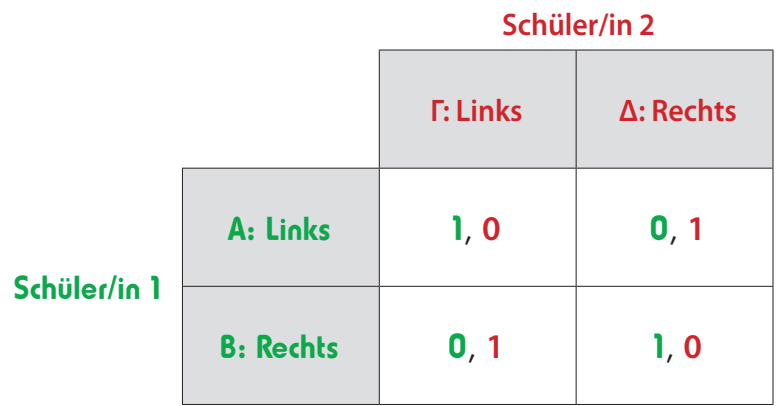

Abb. 8: Beispiel für „Kopf oder Zahl mit zwei Münzen“ 


\begin{tabular}{|c|c|c|c|c|}
\multicolumn{1}{c|}{} & \multicolumn{3}{c}{ Schüler/in 2} \\
\cline { 2 - 5 } \multicolumn{1}{c|}{} & Schere & Stein & Papier \\
\cline { 2 - 5 } & Schere & 0,0 & 0,1 & 1,0 \\
\cline { 2 - 5 } Schüler/in 1 & Stein & 1,0 & 0,0 & 0,1 \\
\cline { 2 - 5 } & Popier & 0,1 & 1,0 & 0,0 \\
\hline
\end{tabular}

Abb. 9: Beispiel für „Schere-Stein-Papier“

- Ein sehr ähnliches Spiel zu Kopf oder Zahl ist Schere-Stein-Papier, obwohl jeder Spieler drei Optionen hat. In diesem Spiel schlägt die Schere das Papier, der Stein die Schere und das Papier den Stein.

- Eigenschaften
- Gleichgewicht: -
- Dominant: -
- Nash-Gleichgewicht: -

\section{Zusammenarbeiten für bessere Ergebnisse?}

In den vorgehenden Beschreibungen gibt es keine Zusammenarbeit zwischen den Spielern. In einigen Spielen wäre es vorteilhaft, wenn sich die Spieler vorher auf eine Option einigen würden. Das trifft für die Spiele Kampf der Geschlechter, Hirschjagd und Gefangenendilemma zu.

Wenn die Option, auf die sich die Spieler geeinigt haben, im Gleichgewicht ist und einmal gewählt wird, gibt es im Nachhinein keinen Grund mehr für die Spieler, von dieser Entscheidung abzuweichen. In zwei Spielen liegt eine solche Option vor:

- Kampf der Geschlechter: A, Г oder B, $\Delta$

- Hirschjagd: А, Г

Eine Einigung auf Zusammenarbeit ist bei einer Hirschjagd am leichtesten zu erzielen (Hirsch jagen, hart arbeiten), weil dadurch beide Spieler das bestmögliche Ergebnis erzielen. Beim Kampf der Geschlechter kann Uneinigkeit über die Wahl des Gleichgewichtes entstehen (Oper oder Boxwettkampf, GW oder Mathe lernen). Beim Gefangenendilemma ist eine Einigung auf A, $\Gamma$ zwar möglich, die Spieler erzielen aber bei einer alleinigen Abweichung von dieser Einigung ein besseres Ergebnis (Komplize belasten, petzen).

Bei den weiteren beiden Spielen führt eine Zusammenarbeit nicht zu einem besseren Ergebnis. Beim Deadlock-Spiel ist das Ergebnis ohne Zusammenarbeit optimal, es kann durch Zusammenarbeit kein besseres erreicht werden. Bei Kopf oder Zahl mit zwei Münzen (und Schere-Stein-Papier) kann das Ergebnis auch mit Zusammenarbeit nicht verbessert werden.
Eine Zusammenarbeit ist daher in beiden Fällen nicht im Interesse der Spieler.

Die Schule kann eine wichtige Rolle darin spielen, wie die Ergebnisse einer zwischenmenschlichen Interaktion bewertet werden. Durch den Aufbau von sozialen Fähigkeiten wird Zusammenarbeit positiver und Konkurrenz negativer bewertet. Eine positive Konnotation von Zusammenarbeit und eine negative Bewertung von Konkurrenz verändert die Bewertung der Ergebnisse und möglicherweise auch die Reihung der eigenen Präferenzen. Dann verändert sich die Art des Spiels. Beispielsweise wird eine Hirschjagd zu einem Deadlock-Spiel. Das passiert, wenn die beiden involvierten Schüler/innen ihre Zusammenarbeit schätzen und es negativ bewerten, wenn sie selbst nicht mitarbeiten (z. B. faul sind, während der/die andere Schüler/in an der Arbeit arbeitet). Verändern die Schüler/ innen ihre Bewertungsmaßstäbe dahingehend, dann ist eine Zusammenarbeit bei innovativen Prozessen sowohl in Schule als auch danach wahrscheinlicher.

\section{Unterrichtsidee}

Eine Lehrperson, die sich mit den Grundlagen der beschriebenen Spieltheorie vertraut gemacht hat, kann einfache Spiele mit Schüler/innen im Unterricht umsetzen. Dabei kann die beiliegende Unterrichtsidee verwendet werden. Aber auch bestehende oder neue Ideen können häufig mit Hilfe der Spieltheorie strukturiert und vermittelt werden.

\section{Überblick der Materialien}

Das Unterrichtsmaterial besteht aus folgende Bausteinen: (1) eine Darstellung und ein Übungsbeispiel zum Verstehen der Normalform, (2) eine Erweiterung dieses Übungsbeispiels zum Üben von Reflexivität, Kreativität und Implementivität und (3) jeweils ein Spiel für den Unterricht, basierend auf den innovationsbezogenen Beispielen zu den fünf Spieltypen aus dem theoretischen Teil.

\begin{tabular}{ll}
\hline Spieltyp & Innovation \\
\hline Deadlock & $\begin{array}{l}\text { Das Gründen einer Firma zum Verleihen von } \\
\text { E-Rollern }\end{array}$ \\
\hline $\begin{array}{l}\text { Kampf der } \\
\text { Geschlechter }\end{array}$ & $\begin{array}{l}\text { Das Verwirklichen einer kühlen Zone in der } \\
\text { Straße }\end{array}$ \\
\hline Hirschjagd & Das Entwerfen einer neven Kleidungslinie \\
\hline $\begin{array}{l}\text { Gefangenen- } \\
\text { dilemma }\end{array}$ & $\begin{array}{l}\text { Das Entwickeln eines Impfstoffes gegen ein } \\
\text { neues Virus }\end{array}$ \\
\hline $\begin{array}{l}\text { Kopf und Zahl } \\
\text { mit zwei Münzen }\end{array}$ & $\begin{array}{l}\text { Das Erkämpfen von neue Verhaltensregeln } \\
\text { für die Polizei }\end{array}$ \\
\hline
\end{tabular}

Abb. 10: Übersicht der Spieltypen und

Beispiele zum Thema Innovation 
Die Materialien werden nachher in dieser Reihenfolge vorgestellt. $\mathrm{Zu}$ den jeweiligen Bausteinen werden auch weitere (didaktische) Hinweise gegeben. Im Unterricht können die Bausteine genau in dieser Reihenfolge eingesetzt werden. Zuerst werden aber die mit dem Material verfolgten Lernziele erläutert und wie das Material zur Erreichung dieser Lernziele beitragen kann.

\section{Lernziele}

Die Lernziele sind (1) das selbstständige Bewerten von Ergebnissen, (2) das Üben von Reflexivität, Kreativität und Implementivität, (3) das Erkennen von zwischenmenschlichen Abhängigkeiten, (4) das Fördern von strategischem Denken und (5) das Schätzen von Zusammenarbeit, speziell in einem innovativen Kontext. Diese Ziele schließen natlos bei den Leitvorstellungen sowie die didaktischen Grundsätze in den Lehrplänen an, wobei das Ziel eines selbst organisierenden und mündig handelnden Menschen in Gesellschaft, Wirtschaft, Politik und Umwelt im Vordergrund steht (BMBWF 2018; Chreisky-Höbinger et al. 2019; Hinsch et al. 2014). Im Fach GW können sie zum Beispiel in der Unterstufe in der 3. Klasse (Einblicke in die Arbeitswelt: Erkennen, dass in der Wirtschaft unterschiedliche Interessen aufeinandertreffen) eingebunden werden (BMBWF 2018). Erfahrungsgemäß werden Spiele von Schüler/innen relativ leicht verstanden (Ableitinger \& Hauer-Typpelt 2008). Die Verwendung der innovationsbezogenen Beispiele ermöglicht einen Einsatz in anderen Klassenstufen.

Das selbstständige Bewerten von Ergebnissen erfolgt bei jedem Spiel und fängt schon beim Übungsbeispiel an. Reflexivität , Kreativität und Implementivität werden auch beim Spielen geübt. Ist das erzielte Ergebnis optimal oder kann ich durch andere Entscheidungen das Ergebnis womöglich verbessern (Reflexivität)? Welche durchführbaren Lösungen gibt es, um ein besseres Ergebnis zustande zu bringen (Kreativität)? Wie binde ich meine Mitspieler ein und wie überzeuge ich sie/ihn von meiner Lösung (Implementivität)? Zudem sind Reflexivität, Kreativität und Implementivität in den beispielhaften Innovationen bei allen der fünf Spiele deutlich vorhanden. So ist zum Beispiel die Reflektivität im Hinterfragen vom Verhalten der Polizei, die Kreativität im Entwicklen eines neuen Impfstoffes und die Implementivität im Verwirklichen einer kühlen Zone verankert.

Es wird beim Spielen auch klar, dass das eigene Ergebnis unweigerlich mit der Entscheidung der Mitspieler/in zusammenhängt. Abhängig vom Spieltyp sind im Alleingang die besten Ergebnisse nicht mit Sicherheit erreichbar. In bestimmten Fällen kann nur durch strategisches Denken und der Einbindung der Mitspieler/in (sowie dessen Optionen) das beste Ergebnisse zustande kommen.

\section{Darstellung und Übungsbeispiel zum Verstehen der Normalform}

Damit Schüler/innen die Optionen (Möglichkeiten) und die Folgen einer Entscheidung im Spiel verstehen können, müssen Sie die Normalform in vereinfachter Form lesen können. Eine mögliche Darstellung die dabei helfen kann befindet sich in Abbildung 11. Zudem können Spiele auch im schulischen Kontext angewendet werden (siehe die fünf klassischen Spiele). Die eine oder andere Anwendung kann auch zum Verständnis der Normalform beitragen, weil damit ein klarer Bezug zur Lebenswelt der Schüler/innen hergestellt werden kann.

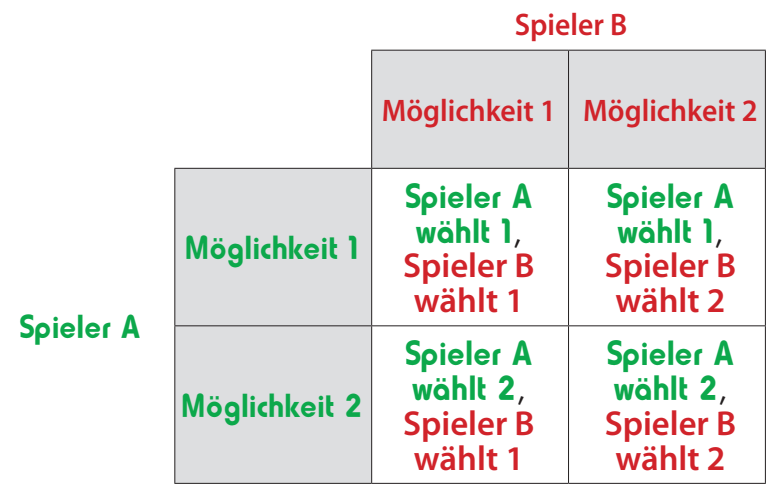

Abb. 11: Übersicht der Ergebnisse für Schüler/innen

Eine Übung zur Festigung dieser Grundlagen ist in Abbildung 12 dargestellt. Ziel dieser Übung ist das Vertraut werden mit der Grundidee der Spieltheorie. Die Schüler/innen befassen sich mit den eigenen Möglichkeiten und den Möglichkeiten der Mitspieler/innen. Das Ergebnis hängt klarerweise mit der Entscheidung beider Schüler/innen zusammen. Es ist sinnvoll, das Spiel in Zweierteams einige Runden lang zu spielen. Dabei beeinflusst die persönliche Bewertung vom Ergebnis und das Verhalten der Mitspieler/ innen die Entscheidungen in den noch kommenden Runden. Genau solches auszuprobieren und auszuloten, stärkt das Verständnis für das Spiel und die Darstellung in einer Normalform.

Melinda und Roberto spielen das Spiel Teilen oder nicht teilen? Sie können dabei Gummibärchen gewinnen. Jede/r Teilnehmer/in entscheidet sich im Geheimen für "Teilen“ oder „Nicht teilen“. Teilen Melinda und Roberto, dann bekommt jeder ein Gummibärchen. Teilt Melinda und Roberto teilt nicht, dann bekommt Roberto zwei Gummibärchen. Teilt Melinda nicht und Roberto teilt, dann bekommt Melinda zwei Gummibären. Teilen weder Melinda noch 


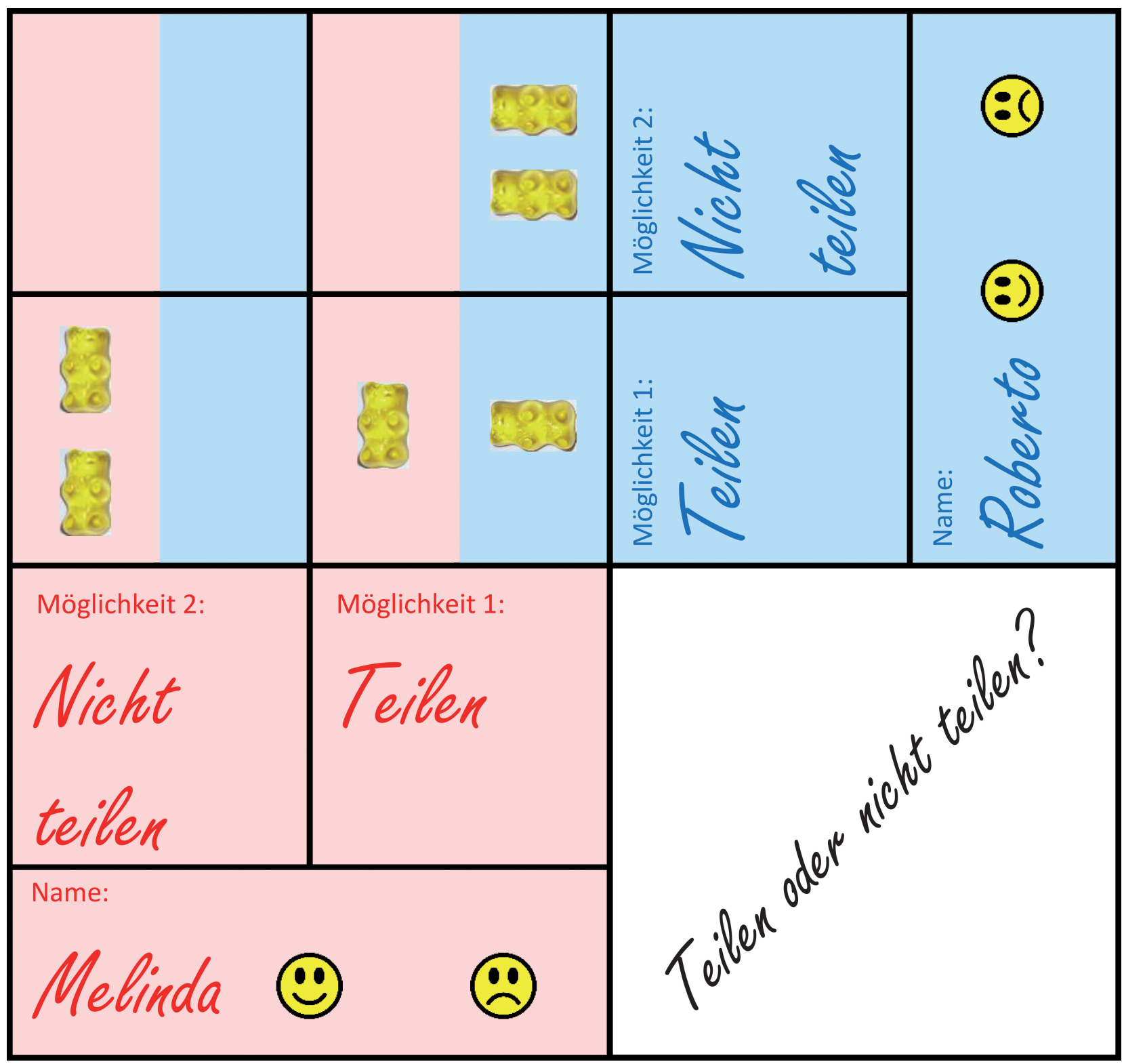

Abb. 12: Beispiel für eine Anwendung des Gefangenendilemmas in der Klasse (eigene Darstellung)

Roberto, dann bekommen sie gar nichts (Abb. 13). Die Schüler/innen kennen diese Möglichkeiten, bevor sie zu spielen beginnen. Es handelt sich bei dem Spiel um eine Form des Gefangenendilemmas, wobei das Nash-Gleichgewicht bedeutet, dass beide nicht teilen und nichts bekommen. Wenn es passiert, dass die Schüler/innen trotzdem teilen, kann die Anzahl

\begin{tabular}{c|c|c|c|}
\multicolumn{1}{c}{} & \multicolumn{2}{c}{ Melinda } \\
\cline { 2 - 4 } Abb. 13 & Teilen & Nicht teilen \\
\cline { 2 - 4 } & Teilen & 1,1 & 0,2 \\
\cline { 2 - 4 } Roberto & Nicht teilen & 2,0 & 1,0 \\
\hline
\end{tabular}

Abb. 13: Ergebnisse wie in Abbildung 10 an Gummibärchen bei der Option „Nicht teilen“ für einen oder beide Spieler erhöht werden (wie in Abbildung 14). Die Lehrperson kann die Ergebnisse des Spiels frei wählen. Es ist selbstverständlich auch möglich etwas anderes als Gummibärchen für die Bewertung der Ergebnisse zu verwenden, wie zum Beispiel Monopoly-Geld.

Abb. 14

\begin{tabular}{|c|c|c|}
\cline { 2 - 3 } \multicolumn{1}{c|}{} & Meilen & Nicht teilen \\
\cline { 2 - 3 } \multicolumn{1}{c|}{ Teilen } & 1,1 & 0,4 \\
\hline Nicht teilen & 4,0 & 0,0 \\
\hline
\end{tabular}

Abb. 14: Verschenkt Roberto Gummibärchen? 


\section{Üben von Reflexivität, Kreativität und Implementivität anhand des Übungsbeispiels}

In einer Erweiterung können beide Spieler zwei Smileys auf ihre persönlichen Ergebnisfelder aufteilen. Ein Smiley steht für eine positive oder negative Bewertung des Ergebnisses. Damit beurteilen die Schüler/innen, welche Ergebnisse sie bevorzugen. Also ob sie die Optionen „Teilen“ oder „Nicht teilen“ besser bewerten würden. Das persönliche Bewerten von Ergebnissen ändert das Ergebnis und das Spiel. Es kann dabei zum Beispiel ein Deadlock-Spiel entstehen. Es müssen nicht zwangsläufig alle Smileys gelegt werden. Hiermit werden Reflexivität (Bewertung der derzeitigen Situation), Kreativität (Erprobung von Lösungen mit Hilfe von Smileys) und Implementivität (gemeinsames Umsetzen der Lösung) geübt.

Wichtig dabei ist, dass übertragbare Ergebnisse (wie z. B. Gummibärchen) und nicht-übertragbare Ergebnisse (wie z. B. einmal den Sessel des Mitschülers/der Mitschülerin auf den Tisch stellen) sich klar unterscheiden. Nur bei übertragbaren Ergebnissen können Spieler nach Abschluss des Spiels eine Neuverteilung der Ergebnisse vornehmen (ein Spieler gibt dem anderen Spieler die Gummibärchen). So ist es in manchen Spielen möglich höhere Gesamtergebnisse zu erzielen. Beim Spiel in Abbildung 11 ist diese Möglichkeit nicht gegeben, weil die Gesamtanzahl von zwei Gummibären in drei der vier Felder gleich ist. Wird diese Gleichwertigkeit zu Gunsten von „Nicht teilen“ verändert wie in Abbildung 12, können beide besser aussteigen, wenn Roberto Melinda zwei Gummibären schenkt (oder vice versa).

\section{Beispiele zum Thema Innovation}

Die fünf folgenden innovationsbezogenen Beispiele sollen hintereinander gespielt werden. Wo dies sinnvoll ist, kann vor dem Spiel in einem kurzen Input zum angesprochenen Thema erfolgen. Es bietet sich an, dass Schüler/ innen alle fünf Spiele in der gleichen Zweiergruppe spielen. Um Verwirrung zu vermeiden, enthält die Normalform nur die eigenen Ergebnisse. In vier der fünf Spiele haben beide Schüler/innen genau die gleichen Optionen und Ergebnisse. Nur beim letzten Spiel (Kopf oder Zahl mit zwei Münzen) hat jede/r Schüler/in eine eigene Perspektive mit eigenen Optionen und Ergebnisse. Jede/r Schüler/in bekommt eine eigene Kopie der eigenen Normalform und markiert im Geheimen die getroffene Entscheidung. Erst dann wird das Ergebnis bestimmt und in der Punktetabelle eingetragen. Zuerst wird ein Durchgang ohne Zusammenarbeit gespielt, also ohne miteinander zu reden. In einer zweiten Runde ist Zusammenarbeit innerhalb der Zweiergruppe erlaubt.

Ziel ist es entweder die eigene Punktezahl und/oder die gemeinsame Punktezahl zu maximieren. Diese Wahl wird den Schüler/innen überlassen. Es ist in der Runde ohne Zusammenarbeit möglich, dass innerhalb der Zweiergruppe beide Ziele verfolgt werden. Die Zielsetzung und die Vorteile der Zusammenarbeit bei verschiedenen Spielen, wird im Plenum diskutiert.

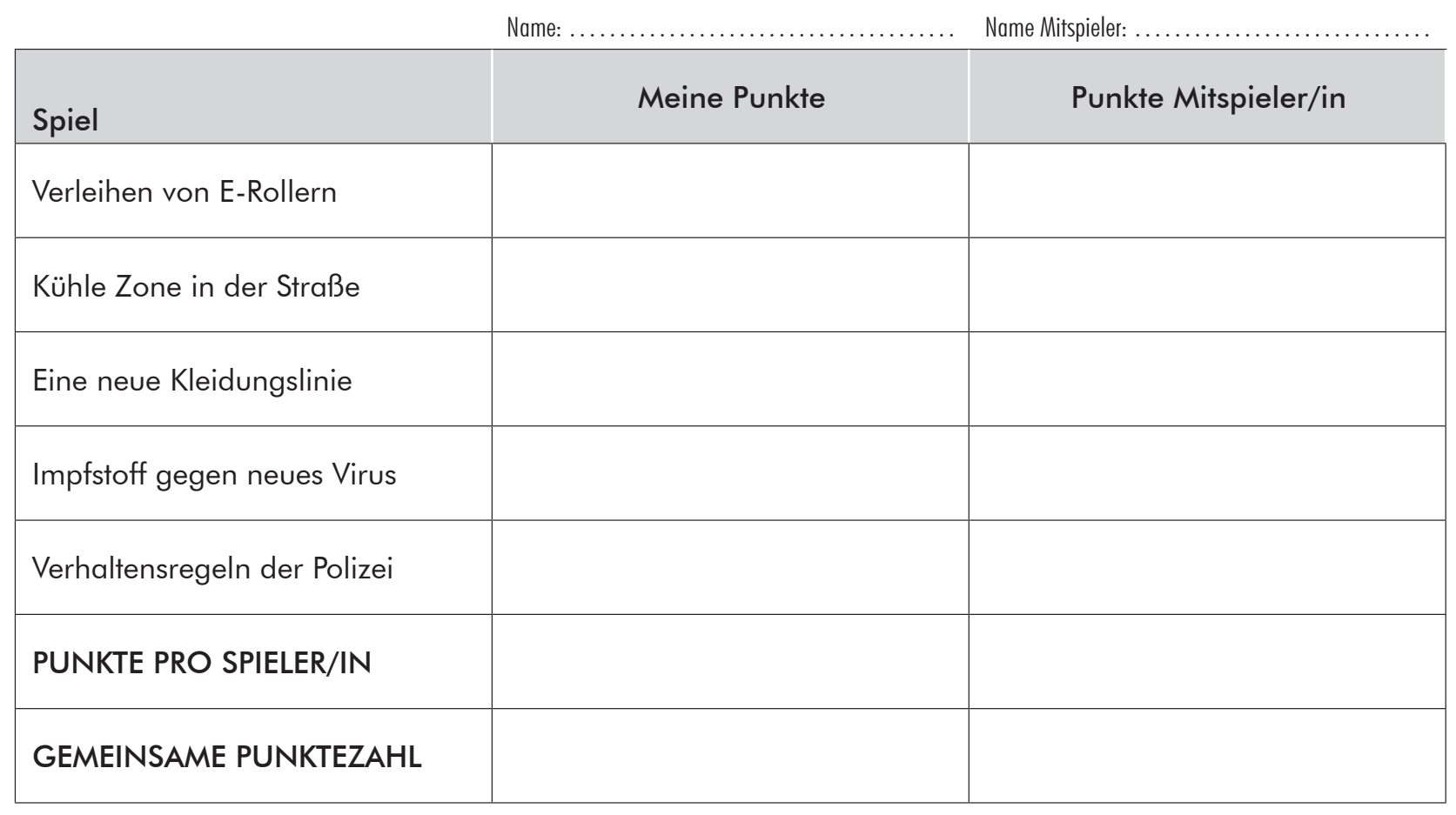




\section{Das Gründen einer Firma zum Verleihen von E-Rollern}

In deiner Heimatgemeinde gibt es viel Autoverkehr. Menschen die mit dem Zug anreisen, kommen nur schwierig dorthin, wo sie hinkommen möchten. Oft muss man auf einen speziellen Bus warten. Danach geht man dann oft immer noch eine lange Strecke zu Fuß. Die andere Möglichkeit ist ein Taxi, was aber viel kostet und den Autoverkehr noch schlimmer macht. Du hast die Idee eine Firma zu gründen und E-Roller zu vermieten. Du weißt, dass noch jemand die gleiche Idee hat. Du musst dich entscheiden, ob du die Firma gründest oder nicht.

\section{MITSPIELER/IN}

\begin{tabular}{|c|c|c|}
\cline { 2 - 4 } & \multicolumn{1}{|c|}{ Gründen } & Nicht gründen \\
\hline Gü̈nden & $\begin{array}{c}\text { Es werden zwei Firmen gegründet. } \\
\text { Beide Firmen verleihen etwa die Hälfte } \\
\text { der E-Roller in der Stadt. Du verdienst } \\
\text { etwas mit deiner Firma. } \\
2 \text { Punkte }\end{array}$ & $\begin{array}{c}\text { Du bist der einzige, der eine Firma zum } \\
\text { Verleihen von E-Roller gegründet hat. Du } \\
\text { verdienst sehr gut mit deiner Firma. }\end{array}$ \\
\hline $\begin{array}{c}\text { Nicht } \\
\text { gründen }\end{array}$ & $\begin{array}{c}\text { Die Firma deines Mitspielers verleiht als } \\
\text { einzige alle E-Roller. Du ärgerst dich } \\
\text { uber die E-Roller im Straßenverkehr, da } \\
\text { sie gefährlich sind und über die E-Roller, } \\
\text { die überall in der Stadt stehen und } \\
\text { rumliegen. } \\
\text { O Punkte }\end{array}$ & $\begin{array}{c}\text { Es wird keine Firma gegründet und es } \\
\text { werden keine E-Roller in der Stadt verlie- } \\
\text { hen. Es gibt keine gefährlichen E-Roller } \\
\text { im Straßenverkehr. Zudem liegen und } \\
\text { stehen keine E-Roller in der Stadt herum. }\end{array}$ \\
1 Punkt
\end{tabular}

\section{Das Verwirklichen einer kühlen Zone in der Straße}

Es soll eine sogenannte kühle Zone in deiner Straße entstehen. Dabei bekommen Fahrräder und Fußgänger mehr Platz, es werden Bäume gepflanzt und Trinkstellen geschaffen. Du findest es toll, dass diese kühle Zone kommen soll. Am meisten gefällt dir daran, dass diese vor deiner Haustür entstehen soll. Ein Nachbar, der einige Häuser weiter wohnt, möchte auch die kühle Zone vor seiner Haustüre. Die kühle Zone wird aber nur dann gebaut, wenn ihr euch einig seid. Du musst dich entscheiden, ob die kühle Zone bei dir vor der Haustür oder vor der Haustür des Nachbarn gebaut werden soll.

\section{MITSPIELER/IN}

\begin{tabular}{|c|c|c|}
\hline & Kühle Zone vor deiner Haustür & $\begin{array}{c}\text { Kühle Zone vor der Haustür des } \\
\text { Nachbarn }\end{array}$ \\
\hline $\begin{array}{l}\text { Kühle Zone } \\
\text { vor deiner } \\
\text { Haustür }\end{array}$ & $\begin{array}{l}\text { Es gibt keine Einigung und deshalb keine } \\
\text { kühle Zone in der Straße. } \\
\text { O Punkte }\end{array}$ & $\begin{array}{l}\text { Die kühle Zone entsteht von deiner } \\
\text { Haustür! } \\
2 \text { Punkte }\end{array}$ \\
\hline $\begin{array}{l}\text { Kühle Zone } \\
\text { vor der } \\
\text { Haustür } \\
\text { des Nach- } \\
\text { barn }\end{array}$ & $\begin{array}{l}\text { Die Kühle Zone entsteht vor der Haustür } \\
\text { des Nachbarn. } \\
1 \text { Punkt }\end{array}$ & $\begin{array}{l}\text { Es gibt keine Einigung und deshalb keine } \\
\text { kühle Zone in der Straße. } \\
\text { 0 Punkte }\end{array}$ \\
\hline
\end{tabular}




\section{Das Entwerfen einer neue Kleidungslinie}

Ihr seid Modedesignerinnen oder Modedesigner. Zu zweit wollt ihr eine neue Kleidungslinie entwerfen, herstellen lassen und in Österreich verkaufen. Ihr habt wirklich tolle neue Ideen. Es muss viel Arbeit geleistet werden, um diese auch erfolgreich umzusetzen. Du musst dich entscheiden, ob du für diese neue Modelinie hart arbeiten möchtest oder faul bist (in der Hoffnung, dass der/die andere hart arbeitet).

\begin{tabular}{|c|c|c|}
\hline & \multicolumn{2}{|c|}{ MITSPIELER/IN } \\
\hline & Hart arbeiten & Faul sein \\
\hline $\begin{array}{c}\text { Hart } \\
\text { arbeiten }\end{array}$ & $\begin{array}{l}\text { Das neue Design der Kleidungslinie ist } \\
\text { sehr toll. Es entsteht ein neuer Trend rund } \\
\text { um eure Kleidung auf Instragram! Die } \\
\text { Kleidung wird von vielen getragen! } \\
3 \text { Punkte }\end{array}$ & $\begin{array}{l}\text { Es entsteht eine mäßig erfolgreiche Klei- } \\
\text { dungslinie. Du verdienst zwar etwas, hast } \\
\text { aber ganz allein hart gearbeitet. Du bist } \\
\text { sehr müde und enttäuscht. } \\
\text { O Punkte }\end{array}$ \\
\hline Faul sein & $\begin{array}{l}\text { Ohne wirklich etwas zu machen, entsteht } \\
\text { eine mäßig erfolgreiche Kleidungslinie. } \\
\text { Du verdienst etwas, ohne dafür gearbei- } \\
\text { tet zu haben. Es gibt Schlimmeres ... } \\
2 \text { Punkte }\end{array}$ & $\begin{array}{l}\text { Es entsteht nicht wirklich eine neue Klei- } \\
\text { dungslinie. Da du nicht viel Arbeit inves- } \\
\text { tiert hast, ist das nicht weiter schlimm. } \\
1 \text { Punkt }\end{array}$ \\
\hline
\end{tabular}

\section{Das Entwickeln eines Impfstoffes gegen ein neues Virus}

Du leitest ein Labor und forschst an einem Impfstoff gegen ein neues Virus. Ein weiteres Labor versucht auch einen Impfstoff zu entwickeln. Wenn ein Labor Zugang zu den Forschungsergebnissen des anderen Labors hat, kann es einen besseren Impfstoff entwickeln. Du musst dich als Laborleiter/in entscheiden, ob du deine Forschungergebnisse mit dem anderen Labor teilen möchtest oder ob du sie lieber geheim hältst.

\section{MITSPIELER/IN}

\begin{tabular}{|c|c|c|}
\cline { 2 - 4 } \multicolumn{1}{c|}{} & Forschungsergebnisse teilen & Forschungsergebnisse geheim halten \\
\hline $\begin{array}{c}\text { For- } \\
\text { schungs- } \\
\text { ergebnisse } \\
\text { teilen }\end{array}$ & $\begin{array}{c}\text { Beide Labore teilen ihre Ergebnisse. Ein } \\
\text { wirksamer Impfstoff wird gemeinsam } \\
\text { entwickelt. }\end{array}$ & $\begin{array}{c}\text { Dein Labor teilt die Ergebnisse. Das } \\
\text { andere Labor hält ihre Ergebnisse } \\
\text { geheim. Das andere Labor entwickelt } \\
\text { einen wirksamen Impfstoff. Du gehst leer } \\
\text { aus! } \\
\text { DU Punkte }\end{array}$ \\
\hline $\begin{array}{c}\text { For- } \\
\text { schungs- } \\
\text { ergebnisse } \\
\text { geheim } \\
\text { halten }\end{array}$ & $\begin{array}{c}\text { Das andere Labor hat seine Ergebnisse } \\
\text { geteilt. Mit deren Hilfe entwickelt dein } \\
\text { Labor allein einen wirksamen Impfstoff. } \\
\text { Du wirst als Held gefeiert! }\end{array}$ & $\begin{array}{c}\text { Beide Labore halten ihre Ergebnisse } \\
\text { geheim. Dein Labor entwickelt einen } \\
\text { Impfstoff der teilwirksam ist. }\end{array}$ \\
\hline
\end{tabular}




\section{Das Erkämpfen von neuen Verhaltensregeln für die Polizei}

Vor einigen Wochen sind erste Videos im Internet erschienen, worauf zu sehen ist, mit wieviel Härte und Gewalt manche Polizisten täglich die Gesetze durchsetzen. Es zirkulieren immer mehr solche Videos im Netz auf sozialen Medien. Es werden in diesen Videos Personen festgehalten, beleidigt und verletzt. Manchmal sterben sogar Personen durch das gewaltsame Auftreten von bestimmten Polizisten. Ein Teil der Bevölkerung geht gegen die Polizei auf die Straße. Sie wollen neue, strenge Verhaltensregeln für Polizisten und harte Strafen, wenn diese von Polizisten gebrochen werden. Die Polizei hält das für eine schlechte Idee. Es wurden zwar Fehler gemacht, die Möglichkeit, wenn nötig, Gewalt einsetzen zu können, ist aber notwendig, um gute Arbeit leisten zu können. Bei den Protesten greifen einige Personen Polizisten an oder zerstören Eigentum der Stadt oder anderer Menschen.

\begin{tabular}{|c|c|c|c|}
\hline & \multicolumn{2}{|c|}{ MITSPIELER/IN (Protest) } \\
\hline & & Schild mitbringen & Laubbläser mitbringen \\
\hline \multirow{2}{*}{$\begin{array}{c}\text { DU } \\
\text { (Polizei) }\end{array}$} & $\begin{array}{l}\text { Schlagstock } \\
\text { verwenden }\end{array}$ & $\begin{array}{l}\text { Du hast keine Chance gegen die } \\
\text { Protestierenden, die sich mit ihrem } \\
\text { Schild schützen. Du musst zurück in } \\
\text { die Kaserne. Neue Regeln werden } \\
\text { eingeführt. } \\
\text { 0 Punkte }\end{array}$ & $\begin{array}{l}\text { Der Einsatz funktioniert sehr gut. } \\
\text { Ohne wirklich jemanden schlagen zu } \\
\text { müssen, kehren die Protestierenden } \\
\text { heim. Der erfolgreiche Einsatz wird } \\
\text { am nächsten Tag in den Medien } \\
\text { gefeiert. Es werden keine neuen } \\
\text { Regeln eingeführt. } \\
2 \text { Punkte }\end{array}$ \\
\hline & $\begin{array}{l}\text { Tränengas } \\
\text { verwenden }\end{array}$ & $\begin{array}{l}\text { Durch das Tränengas ist der } \\
\text { Protest in kürzester Zeit beendet. } \\
\text { Der erfolgreiche Einsatz wird am } \\
\text { nächsten Tag in den Medien gefeiert. } \\
\text { Es werden keine neuen Regeln } \\
\text { eingeführt. } \\
2 \text { Punkte }\end{array}$ & $\begin{array}{l}\text { Du hast keine Chance gegen die } \\
\text { Protestierenden, die mit Laubbläsern } \\
\text { das Tränengas in deine Richtung } \\
\text { blasen. Du musst zurück in die } \\
\text { Kaserne. Neue Regeln werden } \\
\text { eingeführt. } \\
\text { O Punkte }\end{array}$ \\
\hline
\end{tabular}

\begin{tabular}{|c|c|c|}
\multicolumn{1}{c|}{} & \multicolumn{2}{c}{ MITSPIELER/IN (Polizei) } \\
\cline { 2 - 4 } \multicolumn{1}{c|}{} & $\begin{array}{c}\text { Schlagstock verwenden } \\
\text { Du verteidigst dich sehr erfolgreich } \\
\text { gegen die Polizei. Die Polizei wird } \\
\text { rasch in die Kaserne geschickt. Es } \\
\text { werden neve Regeln angekündigt } \\
\text { und eingeführt. }\end{array}$ & $\begin{array}{c}\text { Tränengas verwenden } \\
\text { Du kannst gegen das Tränengas } \\
\text { nichts tun. Schnell entscheidest du } \\
\text { dich, nach Hause zu gehen. Die } \\
\text { Chance auf neue Regeln ist verloren } \\
\text { gegangen. }\end{array}$ \\
(Protest) & $\begin{array}{c}\text { 2 Punkte } \\
\text { O Punkte }\end{array}$ \\
\hline $\begin{array}{l}\text { Laubbläser } \\
\text { mitbringen }\end{array}$ & $\begin{array}{c}\text { Du kannst dich gegen die Polizisten } \\
\text { mit Schlagstock nicht verteidigen. } \\
\text { Schnell entscheidest du dich nach } \\
\text { Hause zu gehen. Die Chance auf } \\
\text { neue Regeln ist verloren gegangen. } \\
\text { 0 Punkte }\end{array}$ & $\begin{array}{c}\text { Mit dem Laubbläser bläst du die } \\
\text { Tränengaswolke zurück zur Polizei. } \\
\text { Die Polizei wird rasch in die Kaserne } \\
\text { geschickt. Es werden neue Regeln } \\
\text { angekündigt und eingeführt. } \\
\text { 2 Punkte }\end{array}$ \\
\hline
\end{tabular}




\section{$7 \quad$ Fazit}

Die Fähigkeit an interaktiven Innovationsprozessen teilzunehmen ist aus humanistischen, emanzipatorischen und wirtschaftlichen Gründen für Schüler/ innen wichtig. Das Entwickeln von kreativen Denkstrategien und das Aufwerten von Zusammenarbeit spielen dabei eine zentrale Rolle. Die Spieltheorie ist gut geeignet, derartige Interaktionen abzubilden, eigene Bewertungen von Ergebnissen zu überdenken und neue Denkstrategien zu üben. Das Unterrichtsbeispiel erlaubt genau das, indem die Teilbereiche der Innovativität, nämlich Reflexivität, Kreativität und Implementivität, in einem spieltheoretischen Kontext anhand von beispielhaften, innovationsbezogenen Situationen geübt werden können. Das entspricht nur einer der vielen möglichen Anwendungen der Spieltheorie im Unterricht, die weitere Potentiale für Erkenntnisprozesse und Lernmöglichkeiten schafft. Bestehende mathematische Ansätze mit Unterrichtsbeispielen, die auf der Spieltheorie basieren, unterstreichen einige weitere Möglichkeiten und den Erfolg der Spieltheorie in praktischen Unterrichtsumgebungen (Ablinger \& Hauer-Typpelt 2008; Girnat 2010; Lehrer-Online 2020; Schneeberger 2018). Die Spieltheorie und naheliegende Disziplinen wie die Verhaltensökonomie und die Ökonomische Psychologie bieten eine schier unerschöpfliche Quelle an interessanten, lustigen und lebensnahen Gedankenexperimenten, Beispielen und theoretischen Grundlagen für einen Unterricht, der sich dem Entscheidungsverhalten von Menschen widmen will.

\section{Acknowledgement}

Die Publikation dieses Beitrags wurde durch das Projekt Inno_Schools des Landes Salzburg unterstützt.

\section{Literatur}

Ableitinger, C. \& P. Hauer-Typpelt (2008): Spieltheorie im Schulunterricht - kann es das spielen? ÖMG-DidaktikHefte 40. S. 1-12. https://www.oemg.ac.at/DK/Didaktikhefte/2007\%20Band\%2040/VortragAbleitingerHauerTyppelt.pdf (10.08.2020)

BMBWF (2018): Lehrplan der allgemeinbildenden höheren Schulen. https://www.ris.bka.gv.at/GeltendeFassung. wxe? Abfrage $=$ Bundesnormen $\&$ Gesetzesnumm er $=10008568$ (09.08.2020).

Bornemann, S. (2011): Koopertion und Kollaboration. Springer, Wiesbaden.

Chreiska-Höbinger, C., C. Fridrich, S. Hinsch, P. Hofmann, H. Pichler, M. Vorage, T. Jekel, L. Keller \& A.
Koller (2019): Entwurf des Fachlehrplans für den Gegenstand Geographie und Wirtschaftliche Bildung (Stand: 15.11.2019). In: GW-Unterricht 156. S. 74-79.

De Faria, P., F. Lima \& R. Santos (2010): CoOpertion in innovation activities: The importance of Partners. In: Research Policy 39(8). S. 1082-1092.

Dixit, A., S. Skeath \& D. Reiley (2014): Games of Strategy. W. W. Norton \& Company, o. O.

Ebner, H. (2005): Management von Innovationsprozessen in Schulen. In: Berufs- und Wirtschaftspädagogik online 2005(4). S. 1-13.

Franke, N. \& S. Shah (2003): How Communities Support Innovative Activities. In: Research Policy 32(1). S. 157-178.

Franke, N. (2005): Open Source \& Co. In: Albers, S. \& O. Gassmann (Hrsg.), Handbuch Technologie- und Innovationsmanagement. Gabler, Wiesbaden. S. 695-712.

Fudenberg, D. \& J. Tirole (1991): Game Theory. o. O. The MIT Press.

Gibbons, R. (1992): Game Theory for Applied Economists. Princeton University Press, o. O.

Girnat, B. (2010): Mathematik auf der Anklagebank Didaktische Überlegungen zu einem Ausflug in die Spieltheorie. In: Bruder, R. \& A. Eichler (Hrsg.): Modellierung von Erastosthenes bis Google. Franzbecker, Hildesheim. S. 63-74.

Gryl, I. (2013): Alles neu - innovativ durch Geographie und GW-Unterricht? In: GW-Unterricht 131. S. 16-27.

Hinsch, S., H. Pichler, T. Jekel, L. Keller \& F. Baier (2014): Semestrierter Lehrplan AHS, Sekundarstufe II. Ergebnis der ministeriellen Arbeitsgruppe. In: GW-Unterricht 136. S. 51-61.

Jekel, T., N. Ferber \& K. Stuppacher (2015): Innovation vs. innovativeness? Do We Support Our Students in (Re-) Inventing the world? In: GI_Forum 3. S. 373-381.

Kuhn, H. W. \& A. W. Tucker (1950): Contributions to the theory of games. Annals of Mathematical Studies. o. O.

Maier, G. W., D. Frey, S. Schulz-Hardt \& F. C. Brodbeck (2001): Innovation. In: G. Wenninger (Hg.): Lexikon der Psychologie. Spektrum, Frankfurt. S. 264-267.

Michalko, M. (2006): Thinkertoys. A handbook of creative-thinking techniques. Ten Speed Press, Berkeley.

Lakhani, K. \& R. G. Wolf (2003): Why Hackers Do What They Do. In: MIT Working Paper 4425. S. 1-27.

Landry, R., N. Amara \& M. Lamari (2001): Does social capital determine innovation? In: Technological Forecasting \& Social Change 69(7). S. 681-701.

Lehrer-Online (2020): Wirtschaftswissenschaftliche Spieltheorie: eine schülerorientierte Einführung. https://www. lehrer-online.de/unterricht/berufsbildung/wirtschaft/ wirtschaftslehre/unterrichtseinheit/ue/wirtschaftswissenschaftliche-spieltheorie-eine-schuelerorientierte-einfuehrung/ (10.08.2020).

Lerner, J. \& J. Tirole (2002): Some Simple Economics of Open Source. Journal of Industrial Economics 50(2). S. 197-234. 
Love, J. \& S. Roper (2015): SME innovation, exporting and growth: A review of existing evidence. In: International Small Business Journal: Researching Entrepreneurship 33(1). S. 28-48.

Pike, A., A. Rodriguez-Pose \& J. Tomaney (2016): Local and Regional Development. Routledge, o. O.

Pittaway, L., M. Robertson, K. Munir, D. Denyer \& A. Neely (2004): Networking and innovation: a systematic review of the evidence. In: International Journal of Management Reviews 5-6(3-4). S. 137-168.

Rousseau, J.-J. (1755): Discourse on inequality. MarcMichel Rey/ Penguin Classics, o. O.

Scharf, C., S. Schmitz \& I. Gryl (2016): Innovativeness as fresh ground. In: GI-Forum 1. S. 250-261.

Scharf, C., S. Weis \& I. Gryl (2017): Innovative Pupils. In: The European Conference on Education: Official Conference Proceedings. S. 295-313.

Scharf, C., I. Gryl, M. Gamper \& S. Weis (2018): Collaborative Innovating - The Potential of Street Art to Shape Spaces. In: GI_Forum 2. S. 156-167.

Schneeberger, F. (2018): „Häuser auf dem Weg zum Gleichgewicht - Game of Thrones “- Spieltheorie für den Einsatz in der Schule. Diplomarbeit, Universität Wien. http:// othes.univie.ac.at/52129/1/54284.pdf (10.08.2020)

Seitz, S. (2008): Der Lehrer als Innovator von Schule? Verlag Dr. Kovač, Hamburg.

Tadelis, S. (2013): Game Theory: An Introduction. Princeton University Press, o. O.

Von Neumann J. (1928): Zur Theorie der Gesellschaftsspiele. In: Mathematische Annalen 100. S. 295-320.

Von Neumann J. \& O. Morgenstern (1944): Theory of Games and Economic Behavior. Princeton University Press, o. O.
Watson, J. (2013): Strategy: An Introduction to Game Theory. W. W. Norton \& Company, o. O.

Weis, S., C. Scharf, L. Greifzu \& I. Gryl (2017): Stimulating by simulating. In: International Conference on Education: Conference Proceedings 386(1;11).

Zichy, M. (2010), Das humanistische Bildungsideal. In: Schmidhuber, M. (Hg.): Formen der Bildung. Frankfurt am Main. S. 9-42.

\section{Wovon Standardwerke der Spieltheorie:}

Dixit, A., S. Skeath \& D. Reiley (2014): Games of Strategy. W. W. Norton \& Company, o. O.

Fudenberg, D. \& J. Tirole (1991): Game Theory. o. O. The MIT Press.

Gibbons, R. (1992): Game Theory for Applied Economists. Princeton University Press, o. O.

Tadelis, S. (2013): Game Theory: An Introduction. Princeton University Press, o. O.

Von Neumann J. \& O. Morgenstern (1944): Theory of Games and Economic Behavior. Princeton University Press, o. O.

Watson, J. (2013): Strategy: An Introduction to Game Theory. W. W. Norton \& Company, o. O.

\section{Bildquellen (11.08.2020)}

Gummibärchen: https://pixabay.com/photos/gummi-bears-gummi-bear-bear-8551/

Smiley glücklich: https://pixabay.com/de/vectors/smileyemoticon-glücklich-gesicht-1635449/

Smiley unglücklich: https://pixabay.com/de/vectors/smiley-emoticon-glücklich-gesicht-1635448/ 